\title{
Pulmonary multifocal arteriovenous malformations lead to ischemic stroke in young adults: a case report and literature review
}

\author{
Xuanfei Jiang ${ }^{1}$, Lifeng $\mathrm{He}^{1}$, Bin Shen ${ }^{2}$, Honggang Ma ${ }^{1}$, Bing Zhang ${ }^{1}$ \\ ${ }^{1}$ Department of Neurology, Huzhou Cent Hospital, Affiliated Cent Hospital Huzhou University, Huzhou, China; ${ }^{2}$ Department of Interventional \\ Surgery, Huzhou Cent Hospital, Affiliated Cent Hospital Huzhou University, Huzhou, China \\ Correspondence to: Bing Zhang. Department of Neurology, Huzhou Cent Hospital, Affiliated Cent Hospital HuZhou University, Huzhou, China. \\ Email: sjnkhz@163.com.
}

\begin{abstract}
In recent years, the incidence of ischemic stroke in young people has gradually increased. The etiology and risk factors of young patients with ischemic stroke are complex, including migraine with aura, inherited thrombophilias, hyperhomocysteinemia, cardiovascular risk factors and malignancy. Pulmonary arteriovenous malformation (PAVM) is a rare cause of ischemic stroke in young people, which can easily be misdiagnosed and missed due to the lack of specific biomarkers. In this case report, we described a 17-year-old stroke patient who was admitted to hospital due to sudden speech failure accompanied by weakness of the right limb, and was diagnosed with cerebral embolism in the emergency department. After mechanical thrombectomy, the blood vessels were completely recanalized and the symptoms of nerve defects disappeared. Subsequent chest CT examination revealed pulmonary vascular malformations and further pulmonary angiography revealed multiple PAVMs in the lungs. Furthermore, spring coil embolization treatment was performed on 2 PAVMs with an inflow greater than $2 \mathrm{~mm}$. Three months later, enhanced chest CT reexamination showed no recanalization of the malformed vessels at the pulmonary embolism site. Therefore, PAVM should be further not excluded for young stroke patients with unknown causes to avoid misdiagnosis and missed diagnosis, interventional embolization is the best treatment method after diagnosis.
\end{abstract}

Keywords: Pulmonary arteriovenous malformation (PAVM); cerebral embolism; mechanical thrombectomy; digital subtraction angiography (DSA); case report

Submitted Sep 18, 2021. Accepted for publication Nov 09, 2021.

doi: 10.21037/apm-21-3034

View this article at: https://dx.doi.org/10.21037/apm-21-3034

\section{Introduction}

Every year, nearly 2 million young people around the world suffer from stroke, which brings serious harm and financial burden to patients and their families. While the incidence of stroke in the elderly has decreased year by year, the incidence of stroke in the young has shown an increasing trend $(1,2)$. The etiology of ischemic stroke in young people is complex, and there are often no traditional risk factors (such as hypertension, hyperlipidemia, diabetes, etc.). The common causes of ischemic stroke may be early onset atherosclerosis, cardiac embolism caused by patent foramen ovale, arterial dissection, and cerebral vasospasm (3). Pulmonary arteriovenous malformation (PAVM) is the abnormal anastomosis of the pulmonary artery and pulmonary vein, and the embolus passes through the abnormal pathway to the systemic circulation, leading to the occurrence of cerebral embolism events, which is a rare cause of ischemic stroke, especially in young people. Most of the previous reports were of single PAVMs. Here, we reported a successfully treated young stroke patient with multiple PAVMs. We present the following article in accordance with the CARE reporting checklist (available at https://dx.doi.org/10.21037/apm-21-3034). 


\section{Case presentation}

The patient, a 17-year-old female student, was admitted to hospital with "sudden speech failure accompanied by right limb weakness for $1.5 \mathrm{~h}$ ". The patient suddenly fell ill at about 17:00 and arrived at the department of emergency of our hospital at 17:40. Physical examination of the patient showed the following: conscious, motor aphasia, bilateral pupils were equally large and round ( $3 \mathrm{~mm}$ in diameter), sensitive light reflex, free eye movements, bilateral frontal line was symmetrical, tongue tilted to the right, right side limb muscle strength level 0 , left side limb strength level 5, reduced muscle tension on the right side, hypoalgesia in the right limb, right Babinski sign positive, left Babinski sign negative, Brudzinski's sign negative, Kernig sign negative, and unable to cooperate with fraternal movement examination. Her National Institute of Health stroke scale (NIHSS) score was 11. Emergency blood routine examination, coagulation function, renal function, electrolytes, and troponin I were normal. Random blood glucose in the emergency department was $6.4 \mathrm{mmol} / \mathrm{L}$. Color doppler echocardiography showed no obvious abnormality in emergency. At 17:51, cerebral artery CT angiography (CTA) showed a left middle cerebral artery embolism. At 18:11, cranial magnetic resonance imaging (MRI) revealed an acute cerebral infarction beside the left lateral ventricle body area and in the left external capsule area. Intravenous thrombolytic therapy was not performed because the patient was not old enough for intravenous thrombolytic therapy. Digital subtraction angiography (DSA) further confirmed embolism in the M1 segment of the left middle cerebral artery (Figure $1 A$ ). At the same time, mechanical thrombectomy was performed under local anesthesia. Puncture of the right femoral artery was completed at 19:03, and the occluded vessel was completely recanalized at 19:57 (Figure 1B). The TICI grade was III. Neurological examination was performed immediately after surgery, which showed the following: conscious, fluent in speech, answered to the point, symmetrical bilateral nasolabial sulcus, muscle strength of extremities level 5, symmetrical bilateral sensory function, bilateral Babinski sign negative, and NIHSS 0 . Diffusion weighted magnetic resonance imaging (DWI) revealed infarction in the external capsule area $24 \mathrm{~h}$ after surgery, with no significant impact on the patient (Figure 1C).

For the mechanical thrombectomy procedure, the patient was supine, routine disinfection was performed, and the surgical drape was laid. Lidocaine local anesthesia was administered, the right femoral artery was punctured with Seldinger's method, and the $8 \mathrm{~F}$ artery sheath was inserted. The pigtail catheter was inserted into the ascending aorta under the guidance of the guiding wire, and upper arch angiography was performed, which indicated that the M1 segment of the left middle cerebral artery was occluded. The catheter was withdrawn, the 8-F guiding catheter was guided by a $5 \mathrm{~F}-125 \mathrm{~cm}$ MPA catheter into the $\mathrm{C} 1$ segment of the left internal carotid artery, and was introduced into the NAVEIN middle catheter in the sponge segment by the coaxial technique. The microcatheter was successfully guided by the 0.014 microguide wire into the M2 segment of the left middle cerebral artery, and the microguide wire was withdrawn. After microcatheter angiography, it was found that the occluded distal vessel had unobstructed blood flow. The SolitaireAB stent $(4 \mathrm{~mm} \times 20 \mathrm{~mm})$ was delivered in place, and the stent was released at the thrombosis site. The observation of the guidance tube angiography confirmed that the blood flow of the left middle cerebral artery was fluent, and the stent remained open for $5 \mathrm{~min}$. Subsequently, both the microcatheter and the stent were withdrawn for thrombectomy, and the assistant used a $50 \mathrm{~mL}$ syringe at the "Y" shape valve of the guiding catheter to form blood reflux to prevent distal embolism. The embolus was pulled twice, angiography indicated vascular recanalization, TICI grade was III, and the operation was completed.

Examination during admission showed that thyroid function, complete set of autoantibodies, anti-cardiolipin antibody, glycosylated hemoglobin, and tumor markers were normal. The 24-h dynamic electrocardiogram showed no abnormality. Ultrasonography of the upper abdomen showed no obvious abnormality in the liver, bile, pancreas, and spleen. Brain CT scan showed no obvious abnormalities, and cerebrovascular MRA showed no abnormalities. Transesophageal three-dimensional echocardiography showed no obvious abnormality. Head and chest plain CT scans showed tortuous vessels in the upper lobe of the left lung. Vascular malformation was considered. Pulmonary artery CTA showed a left PAVM. Pulmonary angiography showed multiple (diffuse) PAVMs in both lungs (Figure 2A). At the same time, spring coil embolization was performed with an inflow greater than $2 \mathrm{~mm}$ (Figure 2B). The patient recovered well and was discharged 3 days later. Three months after the operation, the patient was followed up in the outpatient department. Pulmonary artery CTA showed no abnormal vessels at the embolization site, indicating that the abnormal pathway had been embolized (Figure 2C).

For the PAVM embolization procedure, the patient was 

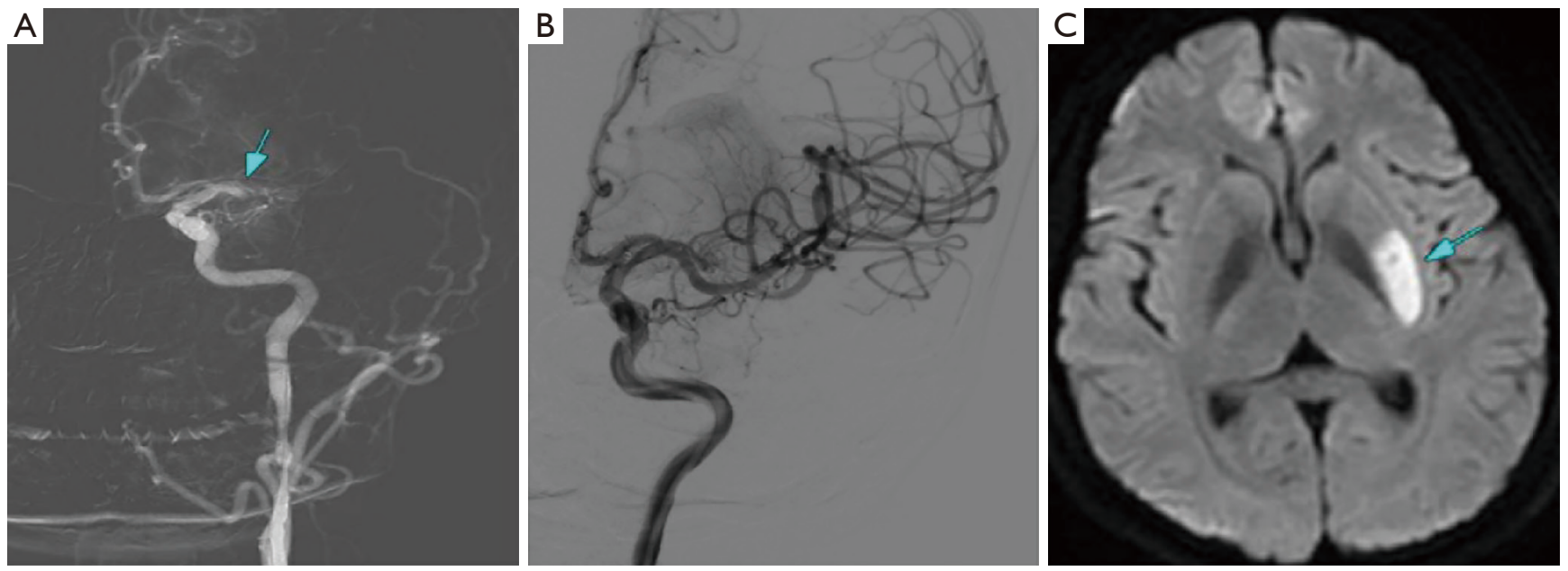

Figure 1 Cerebral embolism and treatment. (A) DSA showed embolism in the M1 segment of the left middle cerebral artery, the arrows represent embolism; (B) the left middle cerebral artery was recanalized after thrombectomy; (C) 24 h after thrombectomy, DWI showed infarction in the external capsule area, the arrows represent infarction area. DWI, diffusion weighted magnetic resonance imaging; DSA, digital subtraction angiography.
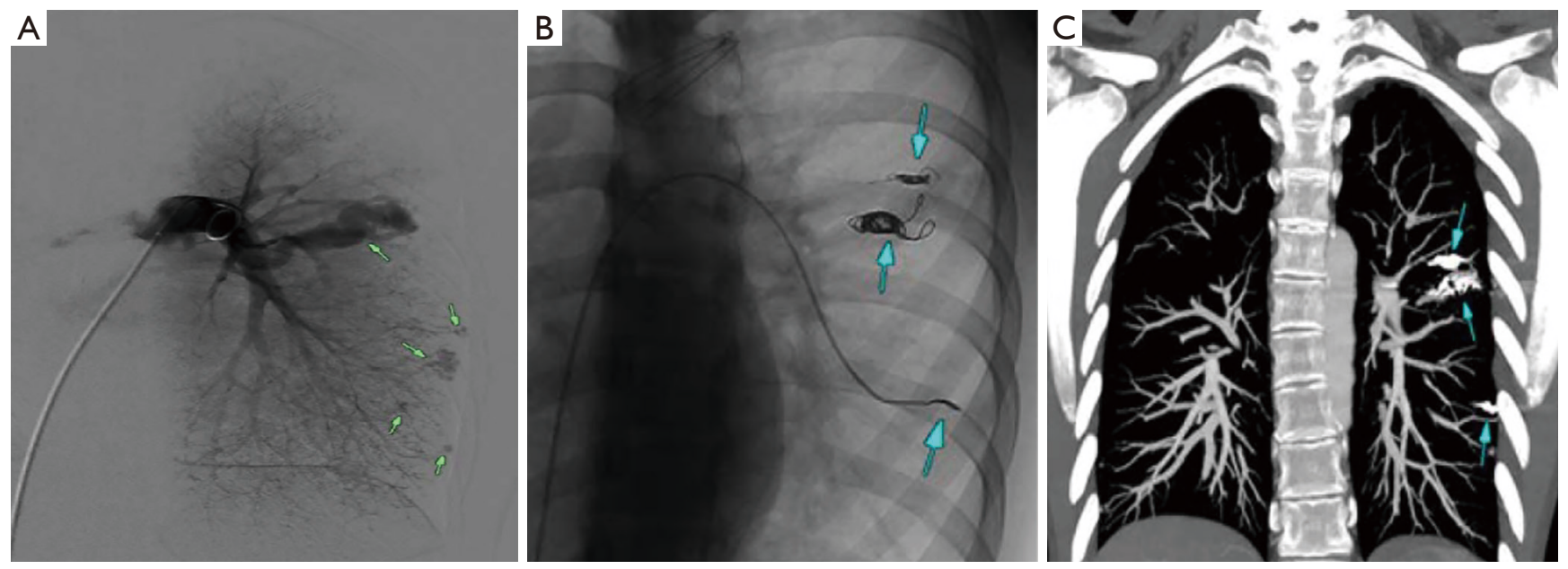

Figure 2 Patient PAVMs and treatment. (A) Pulmonary angiography showed multiple PAVMs, the arrows represent arteriovenous malformations; (B) spring coil embolization was performed for PAVMs with inflow channels larger than $2 \mathrm{~mm}$, the arrows represent spring coil; (C) pulmonary CTA was reviewed 3 months after surgery, the arrows represent pulmonary artery malformation after embolization. PAVMs, pulmonary arteriovenous malformations; CTA, CT angiography.

supine, routine disinfection was performed, and the surgical drape was laid. Lidocaine local anesthesia was administered, the right femoral vein was punctured with Seldinger's method, and a $7-\mathrm{F}$ vein sheath was inserted. The $5-\mathrm{F} \times 70 \mathrm{~cm}$ sheath was then inserted into the main pulmonary artery through the right atrium with the $\mathrm{H} 1$ catheter as the inner sheath. The angiography catheter was taken to the main left pulmonary artery. The angiography showed multiple PAVMs in the left pulmonary artery. The larger was in the left upper lung, and the 2 sites in the inflow duct were larger than $2 \mathrm{~mm}$. A 4-F single-bent catheter was taken to each inflow channel, and 3 interlock controllable spring coils and 1 free coil were taken to embolize the 2 PAVM inflow channels larger than $2 \mathrm{~mm}$ by means of a microcatheter. After embolization, angiography showed amputation change of the inflow arteries, while the PAVM sac and drainage vein were not developed. The catheter 
and catheter sheath were withdrawn, and the operation was completed. The patient returned safely to the ward.

All procedures performed in studies involving human participants were in accordance with the ethical standards of the institutional and/or national research committee(s) and with the Helsinki Declaration (as revised in 2013). Written informed consent was obtained from the parents/ guardian of the patient for publication of this case report and accompanying images. A copy of the written consent is available for review by the editorial office of this journal.

\section{Discussion}

PAVM is the direct communication between the pulmonary artery and pulmonary vein by dilating tortuous blood vessels or hemangiomas. Pulmonary artery blood flows directly into the pulmonary venous circulation without filtering through the pulmonary capillary bed, resulting in increased physiological right-to-left shunt $(4,5)$. The most common cause of idiopathic PAVM is hereditary hemorrhagic telangiectasia (HTT). HTT is an autosomal dominant genetic disorder, the most common of which involves mutations of the ENG and ACVRL1 genes. A total of $62 \%$ of ENG mutants and $10 \%$ of ACVRL1 mutants develop PAVM $(6,7)$. HTT is characterized by arteriovenous malformation, and its diagnosis is based on the Curacao criteria, including telangiectasia, epistaxis, family history, and visceral lesions. If 3 of the criteria are met, the diagnosis of HHT can be established $(8,9)$. Secondary PAVM includes trauma, infection, long-term cirrhosis, mitral stenosis, and other factors (10).

The neurological complications of PAVM include migraine, transient ischemic attack, ischemic stroke, brain abscess, and epilepsy, among others. The incidence of ischemic stroke is $10-18 \%$, the incidence of transient ischemic attack is $6.5-37 \%$, and the incidence of brain abscess is about $6.8 \%(11,12)$. A follow-up study of 143 PAVM patients showed that one-third of those diagnosed with PAVM had a history of ischemic stroke or transient ischemic attack (13). It has been proven that multiple PAVM lesions result in a higher incidence of ischemic stroke than single PAVM lesions (14). The possible pathogenesis involves abnormal venous drainage, which bypasses the capillary bed's filtering capacity and allows bacteria and thrombus to pass directly from venous blood to arterial blood. The formation of thrombus may be caused by hypoxemia resulting from pulmonary artery blood flowing directly into the pulmonary vein without oxygenation, resulting in increased blood viscosity and tendentiousness to thrombosis $(15,16)$. In this young patient, thrombosis dislodged the tortuous vessels to the pulmonary vein and returned to the left atrium, embolizing the left middle cerebral artery via the left ventricle.

The imaging examinations of PAVM mainly include chest CT, enhanced chest CT, and pulmonary angiography, which is the gold standard for the diagnosis of PAVM $(17,18)$. In this case, chest CT plain scan found suspicious lesions, and further enhanced chest CT and pulmonary artery angiography were performed, which helped us to determine the location of the lesions, determine the structure of the supplying arteries and draining veins, and guide the catheter embolization of the lesions. In order to prevent symptom progression and related complications of PAVM patients, interventional embolization is the best treatment method after diagnosis (19). In this case, a transcatheter spring coil was used. The main complication of embolization is benign pleurisy chest pain, which can be treated with nonsteroidal anti-inflammatory drugs (20). The patient had no discomfort after embolization and was discharged after 3 days of observation. In patients with unsuccessful embolization or renal insufficiency, surgical treatment may be considered.

\section{Conclusions}

PAVM has low incidence, lacks clinical specificity, and can easily be missed and misdiagnosed. The possibility of PAVM should be paid attention to in young stroke patients. Lifelong follow-up is required for diagnosed patients and first-degree relatives.

\section{Acknowledgments}

Funding: This work was financially supported by the Public Welfare Technology Application Research of Huzhou Science and Technology Bureau (No. 2018GYB07).

\section{Footnote}

Reporting Checklist: The authors have completed the CARE reporting checklist. Available at https://dx.doi. org/10.21037/apm-21-3034

Conflicts of Interest: All authors have completed the ICMJE uniform disclosure form (available at https://dx.doi. org/10.21037/apm-21-3034). The authors have no conflicts of interest to declare. 
Ethical Statement: The authors are accountable for all aspects of the work in ensuring that questions related to the accuracy or integrity of any part of the work are appropriately investigated and resolved. All procedures performed in studies involving human participants were in accordance with the ethical standards of the institutional and/or national research committee(s) and with the Helsinki Declaration (as revised in 2013). Written informed consent was obtained from the parents/guardian of the patient for publication of this case report and accompanying images. A copy of the written consent is available for review by the editorial office of this journal.

Open Access Statement: This is an Open Access article distributed in accordance with the Creative Commons Attribution-NonCommercial-NoDerivs 4.0 International License (CC BY-NC-ND 4.0), which permits the noncommercial replication and distribution of the article with the strict proviso that no changes or edits are made and the original work is properly cited (including links to both the formal publication through the relevant DOI and the license). See: https://creativecommons.org/licenses/by-nc-nd/4.0/.

\section{References}

1. Boot E, Ekker MS, Putaala J, et al. Ischaemic stroke in young adults: a global perspective. J Neurol Neurosurg Psychiatry 2020;91:411-7.

2. Ekker MS, Boot EM, Singhal AB, et al. Epidemiology, aetiology, and management of ischaemic stroke in young adults. Lancet Neurol 2018;17:790-801.

3. Hankey GJ. Stroke. Lancet 2017;389:641-54.

4. Tellapuri S, Park HS, Kalva SP. Pulmonary arteriovenous malformations. Int J Cardiovasc Imaging 2019;35:1421-8.

5. Kramdhari H, Valakkada J, Ayyappan A. Diagnosis and endovascular management of pulmonary arteriove-nous malformations. Br J Radiol 2021;94:20200695.

6. Kühnel T, Wirsching K, Wohlgemuth W, et al. Hereditary Hemorrhagic Telangiectasia. Otolaryngol Clin North Am 2018;51:237-54.

7. Kritharis A, Al-Samkari H, Kuter DJ. Hereditary hemorrhagic telangiectasia: diagnosis and management from the hematologist's perspective. Haematologica 2018;103:1433-43.

8. Abston E, Hon S, Rodriguez-Lopez J, et al. Treatment of pulmonary hypertension in patients with Hereditary Hemorrhagic Telangiectasia - A case series and systematic review. Pulm Pharmacol Ther 2021;68:102033.
9. Wong HH, Chan RP, Klatt R, et al. Idiopathic pulmonary arteriovenous malformations: clinical and imaging characteristics. Eur Respir J 2011;38:368-75.

10. Kroon S, van den Heuvel DAF, Vos JA, et al. Idiopathic and hereditary haemorrhagic telangiectasia associated pulmonary arteriovenous malformations: comparison of clinical and radiographic characteristics. Clin Radiol 2021;76:394.e1-8.

11. Cartin-Ceba R, Swanson KL, Krowka MJ. Pulmonary arteriovenous malformations. Chest 2013;144:1033-44.

12. Shovlin CL. Pulmonary arteriovenous malformations. Am J Respir Crit Care Med 2014;190:1217-28.

13. Pollak JS, Saluja S, Thabet A, et al. Clinical and anatomic outcomes after embolotherapy of pulmonary arteriovenous malformations. J Vasc Interv Radiol 2006;17:3544; quiz 45.

14. Wozniak L, Mielczarek M, Sabiniewicz R. Paradoxical brain embolism in a young man: is it only a patent foramen ovale? Neurol Neurochir Pol 2015;49:61-4.

15. Lin G, Jiang P, Lou M. Thrombolysis in Ischemic Stroke Patients with Isolate Pulmonary Arteriovenous Malformations. J Stroke Cerebrovasc Dis 2019;28:e68-70.

16. Liu XH, Yang JM. Management of paradoxical embolism in a patient with coexisting patent foramen ovale and masked pulmonary arteriovenous fistula: A case report. Medicine (Baltimore) 2020;99:e19507.

17. Greffier J, Boccalini S, Beregi JP, et al. CT dose optimization for the detection of pulmonary arteriovenous malformation (PAVM): A phantom study. Diagn Interv Imaging 2020;101:289-97.

18. Saboo SS, Chamarthy M, Bhalla S, et al. Pulmonary arteriovenous malformations: diagnosis. Cardiovasc Diagn Ther 2018;8:325-37.

19. Adachi A, Ohta K, Jahangiri $Y$, et al. Treatment of pulmonary arteriovenous malformations: clinical experience using different embolization strategies. Jpn J Radiol 2020;38:382-6.

20. Salibe-Filho W, Piloto BM, Oliveira EP, et al. Pulmonary arteriovenous malformations: diagnostic and treatment characteristics. J Bras Pneumol 2019;45:e20180137.

Cite this article as: Jiang $\mathrm{X}, \mathrm{He} \mathrm{L}$, Shen B, Ma H, Zhang B. Pulmonary multifocal arteriovenous malformations lead to ischemic stroke in young adults: a case report and literature review. Ann Palliat Med 2021;10(11):12034-12038. doi: 10.21037/ apm-21-3034 